\title{
Étude descriptive du processus d'évaluation et de documentation de la douleur postopératoire dans un hôpital universitaire
}

\author{
Dave A Bergeron inf $\mathrm{BSc}^{1}$, Geneviève Leduc $\mathrm{MSc}^{2}$, Serge Marchand $\mathrm{PhD}^{3}$, Patricia Bourgault inf $\mathrm{PhD}^{1}$
}

DA Bergeron, G Leduc, S Marchand, P Bourgault. Étude descriptive du processus d'évaluation et de documentation de la douleur postopératoire dans un hôpital universitaire. Pain Res Manage 2011;16(2):81-86.

Plusieurs études démontrent que les patients reçoivent souvent un traitement insatisfaisant de la douleur en contexte postopératoire. Le but de la présente étude descriptive était d'examiner et d'analyser diverses données ayant trait au soulagement de 40 patients après une intervention chirurgicale non urgente. Les patients ont rempli un journal de douleur afin d'évaluer le niveau d'intensité et de désagrément engendré par la douleur durant les trois premières journées postopératoires. Une analyse du dossier a permis de vérifier la documentation de l'évaluation de la douleur par le personnel infirmier. Les résultats indiquent que la douleur en contexte postopératoire est peu et inadéquatement évaluée et peu documentée par le personnel infirmier. Lorsque la douleur est évaluée selon une échelle numérique, les infirmières ont tendance à la sous-évaluer en comparaison avec l'évaluation notée par les patients. Pour la première journée postopératoire, l'intensité moyenne de la douleur documentée par les infirmières est de $1,57( \pm 0,23)$ sur une échelle numérique de 0 à 10 , alors que l'intensité moyenne documentée par les patients est de 3,82 ( $\pm 0,41)$. Cette étude fait ainsi ressortir qu'il n'y a pas de corrélation significative entre l'intensité de la douleur documentée par l'infirmière et celle notée par le patient, ce qui peut expliquer en partie un soulagement non optimal.

\author{
Descriptive study of the postoperative pain \\ assessment and documentation process in a \\ university hospital
}

Several studies have shown that patients often receive inadequate treatment of postoperative pain. The aim of the present descriptive study was to examine and analyze various data related to the postoperative pain assessment of 40 patients who underwent elective surgery. Pain journals were to be completed by patients during every waking hour for the first three postoperative days to assess both pain intensity and pain unpleasantness. A post hoc analysis of patient records permitted verification of pain assessment by nurses for each patient. The results showed that not only was postoperative pain rarely assessed using a valid scale, it was also poorly documented. In addition, when nurses assessed and documented postoperative pain using a numerical scale, their results were very different from patients' assessments. For the first postoperative day, the mean $( \pm \mathrm{SD})$ pain intensity documented by nurses on a 0 to 10 numerical scale was $1.57 \pm 0.23$, while the mean pain intensity noted by patients using the same scale was $3.82 \pm 0.41$. Statistical analysis showed that there was no significant correlation between mean pain intensity documented by nurses and the mean pain intensity noted by patients.

Mots clés : Centre hospitalier; Douleur postopératoire; Évaluation; Infirmières

\footnotetext{
T a douleur en contexte postopératoire est un enjeu important (1)

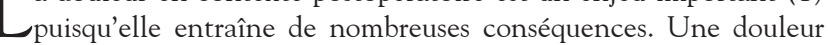
non soulagée constitue un stress physiologique néfaste (2) engendrant l'augmentation de la fréquence cardiaque et de la pression artérielle, le ralentissement de la vidange gastrique, des déséquilibres endocriniens et une diminution de la capacité respiratoire $(3,4)$. L'immobilité résultante de la douleur favorise l'apparition de thrombose veineuse profonde et d'embolie pulmonaire $(1,2,4-6)$. Des répercussions psychologiques sont possibles, telles une augmentation de l'anxiété, l'apparition de troubles du sommeil, la fatigue, l'agitation, l'irritabilité, l'agressivité et, surtout, la présence de souffrance et de détresse émotionnelle (4,7-9). Les personnes ayant un niveau de douleur postopératoire élevé sont également plus susceptibles de présenter un délirium $(10,11)$. Toutes ces complications auront pour ultime conséquence de prolonger inutilement la durée du séjour du patient en milieu hospitalier $(1,3,12)$ et même de voir la douleur se chroniciser $(8,13-17)$. Compte tenu de toutes les répercussions d'une douleur postopératoire peu ou inadéquatement soulagée, il est primordial d'en assurer la gestion efficace.

Or, une gestion efficace sous-tend une évaluation régulière de la douleur $(18,19)$. Cette évaluation est habituellement dévolue aux infirmières. À ce titre, entre 1992 et 2001, on a observé une augmentation du nombre d'évaluations de la douleur faites par les infirmières

en postopératoire, sans diminution significative de l'intensité de la douleur ressentie par les patients (20). Pour évaluer la douleur, les infirmières utilisent très peu les outils d'évaluation, telles les échelles d'autoévaluation. Elles recourent plus souvent à une évaluation narrative (question non systématique) $(21,22)$. De plus, il existe une différence marquée entre l'évaluation de la douleur effectuée par l'infirmière et celle fournie par le patient (23). La documentation de l'évaluation et du traitement de la douleur dans les notes d'observation des infirmières semble également problématique, car le personnel infirmier l'effectue de façon aléatoire et non systématique (24-26).

De nombreuses barrières peuvent expliquer que l'évaluation de la douleur demeure sous-optimale. Ces dernières concernent à la fois les patients, les professionnels de la santé et l'organisation (27-29). Ainsi, plusieurs patients trouvent normal de ressentir de la douleur après une chirurgie et considèrent qu'il n'est pas nécessaire d'en informer le personnel soignant (30). La crainte et les fausses croyances entourant les opiacés expliquent également l'attitude des patients envers l'expression de leur douleur (29). Chez les professionnels, notamment les infirmières, un manque de connaissances concernant la gestion de la douleur, une non-priorisation du soulagement de la douleur et une évaluation inadéquate de celle-ci sont maintes fois nommés comme éléments explicatifs (27-29). Au sein de l'organisation, l'absence d'uniformisation dans l'utilisation d'outils

${ }^{1}$ École des sciences infirmières de l'Université de Sherbrooke; ${ }^{2}$ Faculté de médecine et des sciences de la santé de l'Université de Sherbrooke; ${ }^{3}$ Centre de recherche Étienne-Lebel du Centre hospitalier universitaire de Sherbrooke, Sherbrooke, Québec

Correspondance: Patricia Bourgault inf PhD, Université de Sherbrooke, Faculté de médecine et des sciences de la santé, École des sciences infirmières, 3001 , $12^{e}$ Avenue Nord, Pavillon Gérald-La Salle, bureau 4028, Sherbrooke, Québec J1H 5N4. Téléphone 819-564-5351, télécopieur 819-820-6816, courriel patricia.bourgault@usherbrooke.ca
} 
d'évaluation valides et la documentation insuffisante de la douleur au dossier sont en cause (24-29).

Certaines études canadiennes ont porté sur la gestion de la douleur postopératoire, mais dans des contextes de soins particuliers, comme c'est le cas auprès des patients ayant subi de graves brûlures ou une angioplastie ou auprès des personnes dans un état critique (3,31-33). Aucune étude canadienne traitant de l'évaluation de la douleur postopératoire auprès de patients adultes hospitalisés dans une unité de chirurgie générale n'a été recensée. Étant donné le nouveau cadre législatif québécois réglementant les professionnels de la santé, les infirmières ont maintenant l'obligation légale d'évaluer l'état global du patient, y compris ses aspects physiques et mentaux. Cette évaluation inclut le monitorage de la douleur (34). Il est donc important de s'attarder aux pratiques des infirmières en regard de l'évaluation de la douleur postopératoire auprès des patients hospitalisés en chirurgie générale afin de mieux connaître l'état de la situation. Les observations obtenues permettront de proposer des stratégies plus ciblées afin d'améliorer cette pratique si elle s'avère inadéquate et de l'adapter à la nouvelle législation.

\section{Objectifs}

L'objectif principal de la présente étude est de décrire la méthode, la fréquence et la documentation de l'évaluation de la douleur effectuée par des infirmières soignantes en contexte postopératoire de chirurgie générale. L'objectif secondaire est de comparer les évaluations de la douleur effectuées par les participants à l'étude à celles notées par leurs infirmières soignantes.

\section{MÉTHODOLOGIE}

\section{Participants}

Un devis descriptif corrélationnel a permis de décrire l'évaluation de la douleur. La présente étude comporte une composante prospective comportant des mesures multiples pour la partie axée sur l'évaluation de la douleur par les patients, ainsi qu'une composante rétrospective pour la seconde partie répertoriant les évaluations de la douleur effectuées par le personnel infirmier. À partir de la liste des chirurgies non urgentes d'un centre hospitalier universitaire tertiaire de 682 lits, un échantillon de convenance de 60 patients a été recruté. Durant cette période, 1731 chirurgies non urgentes nécessitant une hospitalisation ont été réalisées au sein de cet établissement. Les participants ont tous été recrutés en clinique préopératoire et ont tous été hospitalisés dans une unité de chirurgie générale pendant la période de l'étude. Il est important de préciser qu'au moment de l'étude, aucune politique ou directive sur la gestion et l'évaluation de la douleur postopératoire n'avait été mise en place au sein de l'établissement concerné. Les infirmières n'avaient reçu aucune formation particulière sur l'évaluation et le soulagement de la douleur.

Tous les participants devaient être âgés de 18 à 75 ans, subir une chirurgie non urgente et être en mesure d'évaluer régulièrement leur douleur pendant les trois premiers jours de leur hospitalisation. Ils devaient être hospitalisés dans une unité de chirurgie générale pendant la période de l'étude. Les personnes souffrant de maladies pouvant altérer la compréhension et la perception de la douleur et subissant une chirurgie en lien avec un cancer, à l'exception de la prostatectomie, étaient exclues de la présente étude. Les participants ayant subi une prostatectomie étaient inclus dans l'étude puisqu'il n'y a pas de différence significative quant à l'intensité de la douleur postopératoire et à la présence de douleur chronique entre les patients présentant ou non une douleur reliée au cancer $(35,36)$. Le type et la voie d'administration de l'analgésie n'ont pas été pris en compte dans les critères de sélection, car les participants ont été recrutés en préopératoire, alors que leur plan thérapeutique analgésique n'était pas encore établi.

En cours d'étude, 20 sujets ont été retirés de l'étude, car ils n'étaient pas en mesure de remplir adéquatement le journal évaluant leur douleur. La collecte des données le lendemain de la chirurgie explique cette situation, car l'effet de l'anesthésie combiné à celui des analgésiques affectant l'état cognitif empêchait les participants de remplir correctement le journal de douleur.

Le comité d'éthique de l'établissement a approuvé le protocole de recherche, le formulaire de consentement et les instruments de mesure. Les patients savaient que les données recueillies seraient utilisées pour des besoins de recherche. Ils étaient également informés que leur refus de participer à l'étude ne nuirait en rien à la qualité de leurs soins.

\section{Processus}

Journal de douleur : L'évaluation par les participants de leur douleur devait être faite à chaque heure d'éveil lors des trois premières journées postopératoires. La douleur était évaluée en terme d'intensité et d'aspect désagréable, selon une échelle visuelle analogique de 0 (aucune douleur/aucunement désagréable) à 10 (pire douleur imaginable/la plus désagréable possible) et consignée dans un journal de douleur pour chacune des journées postopératoires. Ce journal de douleur permet de mesurer les répercussions de la douleur dans la vie des participants (37) et a été utilisé dans plusieurs études cliniques locales (38-42). Afin de faciliter la collecte de données pour les participants et d'ainsi améliorer le nombre de données recueillies, les patients n'avaient pas à documenter le contexte entourant l'apparition de leur douleur.

En préopératoire, tous les participants ont reçu les mêmes consignes sur la façon d'évaluer leur douleur et de remplir le journal de douleur, lesquelles leur ont été répétées lors de leur première journée postopératoire. On insistait sur le fait que les participants devaient indiquer, lors de chaque heure d'éveil, l'intensité et l'aspect désagréable de leur douleur. On leur expliquait les concepts d'intensité et d'aspect désagréable par l'analogie d'une radio, où l'intensité était comparée au volume et l'aspect désagréable, à l'appréciation de la mélodie (43). Chacun des participants recevait une visite chaque jour afin de s'assurer de leur compréhension, de se faire rappeler les directives et d'optimiser le nombre de données recueillies. C'est lors de ces visites, au premier jour postopératoire, qu'il a été noté que certains patients n'étaient pas en mesure de remplir le journal en raison des anesthésiques. Le journal de douleur ne servait que pour les besoins de l'étude et n'était pas consulté par les infirmières soignantes des participants. Il était placé dans une chemise, de façon à ce que le personnel ne consulte pas l'évaluation inscrite par les participants.

Révision du dossier médical : Un assistant de recherche a également révisé le dossier hospitalier des participants. Les évaluations de la douleur effectuées par les infirmières auprès des participants ont toutes été répertoriées, autant les évaluations narratives que celles réalisées au moyen d'une échelle de mesure. Pour ce faire, les notes d'observation de l'infirmière, les feuilles d'évaluation de la douleur pour les patients sous analgésie contrôlée par le patient (ACP) et sous épidurale ainsi que le dossier informatisé où sont consignés les paramètres fondamentaux, dont l'évaluation de la douleur, ont été consultés. Toutes les évaluations documentées sous forme numérique sur une échelle de 0 à 10 ont été regroupées dans la catégorie «évaluations numériques des infirmières ». Les évaluations descriptives ou narratives, telles que « le patient semble non souffrant » ou « le patient est confortable », ont été regroupées dans la catégorie «évaluations narratives des infirmières". Cette façon de procéder a permis de colliger l'ensemble des évaluations numériques et narratives documentées par les infirmières.

Analyses statistiques : Toutes les données sur les évaluations de la douleur effectuées par les participants et par les infirmières ont été regroupées au sein d'une base de données dénominalisées et classées en fonction du type d'évaluation (évaluations numériques des participants, évaluations narratives des infirmières et évaluations numériques des infirmières), du participant et de l'heure à laquelle elles ont été effectuées. Afin de faciliter l'analyse statistique et la comparaison des évaluations numériques entre elles, une moyenne de l'ensemble des évaluations numériques des patients et des infirmières par jour post-opératoire a ensuite été calculée pour chacun des patients. Les analyses statistiques ont ensuite été réalisées à l'aide du logiciel SPSS 15.0 (Compagnie IBM, États-Unis) et ont été validées 
TABLEAU 1

Données sociodémographiques des participants à l'étude

\begin{tabular}{lc}
\hline & Échantillon $(\mathbf{n}=\mathbf{4 0})$ \\
\hline Âge, années, moyenne ( \pm ÉT) & $54,8( \pm 3,4)$ \\
Homme & $48,5( \pm 2,1)$ \\
Femme & \\
Sexe, $n(\%)$ & $13(32,5)$ \\
Homme & $27(67,5)$ \\
Femme & \\
Chirurgie, n (\%) & $9(22,5)$ \\
Prostatectomie & $20(50)$ \\
Hystérectomie & $4(10)$ \\
Prothèse du genou & $3(7,5)$ \\
Prothèse de hanche & $4(10)$ \\
Autre & $9,3( \pm 3,3)$ \\
Jours d'hospitalisation, moyenne $( \pm$ ÉT) &
\end{tabular}

ÉT Écarte-type

par une statisticienne. Le niveau de signification pour tous les tests statistiques a été fixé à p<0,05.

\section{RÉSULTATS}

Données sociodémographiques des participants à l'étude

L'échantillon final est composé de 40 participants, soit 13 hommes et 27 femmes, ce qui représente $2,3 \%$ de toutes les interventions chirurgicales non urgentes nécessitant une hospitalisation effectuées dans l'établissement durant la période de cette étude (tableau 1). L'âge moyen des participants est de $54,8( \pm 3,4)$ ans pour les hommes et de $48,5( \pm 2,1)$ ans pour les femmes. Les chirurgies subies sont, par ordre d'importance, l'hystérectomie (50\%), la prostatectomie $(22,5 \%)$, l'arthroplastie du genou (10\%) et l'arthroplastie de la hanche (7,5\%). Trois $(10 \%)$ participants ont subi d'autres types de chirurgies non urgentes. Le nombre moyen de jours d'hospitalisation (préopératoire et postopératoire) des participants est de 9,3 $( \pm 3,3)$ jours.

Évaluation de la douleur postopératoire par les infirmières Documentation de la douleur : Ces données statistiques incluent tous les types d'évaluation documentés par les infirmières, dont plusieurs évaluations narratives n'utilisant pas une échelle standardisée. Les résultats du tableau 2 indiquent que, chez 7,5\% des participants, aucune évaluation de la douleur n'a été documentée lors de la première journée postopératoire. Lors de cette même journée, 17,5\% patients ont eu trois évaluations ou moins de leur douleur documentée, alors que $50 \%$ des patients ont eu entre quatre et six évaluations de la douleur documentées dans leur dossier médical. Seulement 27,5\% des patients ont eu plus de six évaluations de la douleur documentées. Lors des journées suivantes, on a observé une diminution du nombre d'évaluations effectuées par les infirmières auprès des participants. Pour les deux journées subséquentes, $12,5 \%$ et $27,5 \%$ des patients n'ont eu aucune évaluation de la douleur documentée et 32,5\% et $45 \%$ des patients ont eu entre une et trois évaluations de leur douleur.

Utilisation de l'échelle numérique : Les résultats qui figurent au tableau 3 ne renferment que les évaluations numériques de la douleur documentées par les infirmières. Le tableau 3 permet de constater que les infirmières utilisent peu les échelles numériques pour évaluer et documenter la douleur des patients en contexte postopératoire, et ce, même si elles doivent remplir une grille d'évaluation standardisée de la douleur pour les participants sous ACP et sous épidurale. En effet, lors de la première journée postopératoire, 34 participants étaient sous $\mathrm{ACP}$ ou sous épidurale, tandis que ce nombre diminuait à 16 et quatre participants pour les jours postopératoires 2 et 3, respectivement. Selon le tableau 3, lors des jours postopératoires 1, 2 et 3, $10 \%, 25 \%$ et $42,5 \%$ des patients n'ont eu aucune évaluation de la douleur au moyen d'une échelle numérique documentée. De plus, 37,5\% des patients ont eu de une à trois évaluations faites à l'aide d'une échelle
TABLEAU 2

Nombre d'évaluations de la douleur narratives et numériques documentées par les infirmières auprès des participants sur une période de $\mathbf{2 4}$ heures pendant les trois premiers jours postopératoires

\begin{tabular}{lccc}
\hline Nombre d'évaluationl & Jour $\mathbf{1}$ & Jour $\mathbf{2}$ & Jour $\mathbf{3}$ \\
\cline { 2 - 4 } 24 heures & Fréquence (\%) & Fréquence (\%) & Fréquence (\%) \\
\hline 0 & $3(7,5)$ & $5(12,5)$ & $11(27,5)$ \\
$1-3$ & $7(17,5)$ & $13(32,5)$ & $18(45,0)$ \\
$4-6$ & $20(50,0)$ & $18(45,0)$ & $11(27,5)$ \\
$7-9$ & $9(22,5)$ & $4(10,0)$ & $0(0,0)$ \\
10 et + & $1(2,5)$ & $0(0,0)$ & $0(0,0)$ \\
Total & 40 & 40 & 40 \\
\hline
\end{tabular}

TABLEAU 3

Nombre d'évaluations de la douleur au moyen d'une échelle numérique documentées par les infirmières auprès des participants sur une période de 24 heures pendant les trois premiers jours postopératoires

\begin{tabular}{lccc}
\hline \multirow{2}{*}{ 24 hombre d'évaluation/ } & Jour $\mathbf{1}$ & Jour $\mathbf{2}$ & Jour 3 \\
\cline { 2 - 4 } & Fréquence (\%) & Fréquence (\%) & Fréquence (\%) \\
\hline 0 & $4(10,0)$ & $10(25,0)$ & $17(42,5)$ \\
$1-3$ & $15(37,5)$ & $22(55,0)$ & $19(47,5)$ \\
$4-6$ & $15(37,5)$ & $7(17,5)$ & $4(10,0)$ \\
$7-9$ & $5(12,5)$ & $1(2,5)$ & $0(0,0)$ \\
10 et + & $1(2,5)$ & $0(0,0)$ & $0(0,0)$ \\
Total & 40 & 40 & 40 \\
\hline
\end{tabular}

numérique lors de leur première journée postopératoire, tandis que $37,5 \%$ des patients en ont eu de quatre à six. Seulement $17,5 \%$ des patients ont subi plus de six évaluations de la douleur au moyen d'une échelle numérique pendant la première journée. Lors des journées suivantes, on observe de nouveau une diminution significative du nombre d'évaluations effectuées par les infirmières auprès des participants.

Comparaison entre les évaluations de la douleur documentées par les participants et celles documentées par le personnel infirmier Le graphique 1 permet de comparer la moyenne des évaluations numériques notées par des patients dans leur journal de douleur avec la moyenne des évaluations numériques documentées par les infirmières pour chacun des trois premiers jours postopératoires. Le premier jour postopératoire, les 40 participants ont évalué leur douleur en moyenne $10,5( \pm 0,8)$ fois dans leur journal, selon une échelle numérique. Le deuxième jour, 36 participants ont fait en moyenne 7,6 $( \pm 0,7)$ évaluations numériques de leur douleur, tandis que 32 participants les ont effectuées en moyenne $8,6( \pm 1,1)$ fois le troisième jour.

Selon les résultats du graphique 1 , lorsque les infirmières évaluent et documentent la douleur à l'aide d'une échelle numérique, la moyenne d'intensité de la douleur de leurs évaluations numériques diffère de la moyenne de l'intensité de la douleur des évaluations faites par les patients dans leur journal. Par exemple, pour une même heure, l'infirmière peut noter la douleur à 2 sur 10 alors que le patient l'a évaluée au même moment à 4 sur 10. Pendant la première journée postopératoire, cette différence est très hautement significative $(p<0,01)$, avec une moyenne d'intensité de la douleur documentée par les infirmières de $1,6( \pm 0,2)$ sur une échelle numérique de 0 à 10 , tandis que la moyenne d'intensité documentée par les patients est de 3,8 $( \pm 0,4)$. Cet écart tend à diminuer lors des jours postopératoires 2 et 3 , mais reste hautement significatif lors de la deuxième journée $(p<0,01)$. L'écart est cependant non significatif $(p=0,084)$ pendant la troisième journée. Il y a donc une différence significative entre la moyenne des évaluations de la douleur documentées par l'infirmière et la moyenne de celles notées 


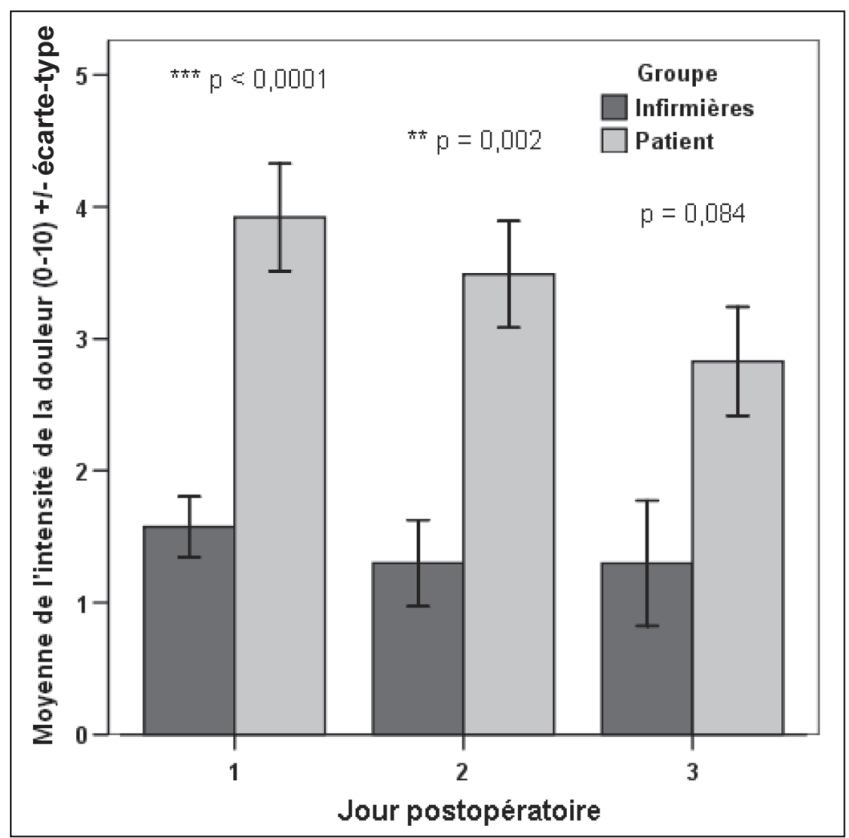

Graphique 1) Comparaison de la moyenne de l'intensité de la douleur notée par les patients dans leur journal selon une échelle numérique de 0 à 10 et de la moyenne de l'intensité de la douleur documentée par les infirmières selon une échelle numérique de 0 à 10 sur une période de 24 heures pendant les trois premiers jours postopératoires

par les patients dans le journal pendant chacune des deux premières journées postopératoires.

Une analyse de corrélation a aussi été réalisée entre la moyenne de l'intensité de la douleur des évaluations documentées à l'aide d'une échelle numérique par les infirmières pour chacun des participants pendant chacune des trois journées postopératoires et la moyenne de l'intensité de la douleur évaluée par les patients dans leur journal de douleur pendant chacune de ces trois journées. Comme l'indique le tableau 4, il n'y a pas de corrélation significative pour chacune des trois premières journées postopératoires entre la moyenne de l'intensité de la douleur des évaluations documentées par les infirmières à l'aide d'une échelle numérique et celle indiquée par les participants dans leur journal de douleur rempli au cours de l'étude.

\section{EXPOSÉ}

En premier lieu, il est important de rappeler que cette étude a été réalisée, en partie, à l'aide du dossier papier et informatique des participants, dans lequel l'infirmière doit documenter les évaluations de la douleur effectuées. Il est donc impossible de savoir avec exactitude comment les infirmières ont effectué leurs évaluations de la douleur auprès des patients, ce qui peut avoir une influence certaine sur la moyenne de l'intensité de la douleur documentée par les infirmières utilisée pour la comparaison avec la moyenne de l'intensité de la douleur notée par les patients dans leur journal de douleur. Il se peut également que la documentation de la douleur par les infirmières ne soit pas totalement représentative de l'évaluation faite par les infirmières auprès de ces mêmes participants. Cependant, en raison d'un possible biais de désirabilité sociale, les autres méthodes de collecte de données, telles que l'observation directe des infirmières ou l'utilisation de questionnaires remplis par les infirmières, auraient probablement été moins représentatives de la situation réelle que la méthode retenue lors de cette étude.

Il est aussi nécessaire de mentionner que le taux élevé d'abandon des participants, soit $30 \%$, peut aussi avoir biaisé la moyenne de l'intensité de la douleur notée par les patients dans leur journal de douleur. Cependant, les patients ont évalué leur douleur toutes les deux à trois heures, ce qui permet d'obtenir un nombre d'évaluations suffisamment important pour valider les résultats recueillis auprès des patients. De
TABLEAU 4

Corrélation entre la moyenne de l'intensité de la douleur notée par les patients dans leur journal selon une échelle numérique de 0 à 10 et celle documentée par les infirmières selon une échelle numérique de 0 à 10 sur une période de $\mathbf{2 4}$ heures pendant les trois premiers jours postopératoires

\begin{tabular}{lcrc}
\hline Journée postopératoire & Nombre de comparaison & $\mathbf{r}$ & $\mathbf{p}$ \\
\hline Jour 1 & 32 & 0,212 & 0,243 \\
Jour 2 & 27 & $-0,222$ & 0,266 \\
Jour 3 & 16 & 0,371 & 0,157 \\
\hline
\end{tabular}

plus, le rappel quotidien des directives aux participants a permis d'augmenter la qualité de leurs évaluations. Le taux élevé d'abandon peut s'expliquer en partie par l'effet de l'anesthésie combiné avec ceux des analgésiques affectant l'état cognitif. D'ailleurs, lors d'études subséquentes similaires, les participants ont rempli le journal de douleur à partir de la deuxième journée postopératoire, ce qui a eu pour conséquence de diminuer le taux d'abandon des participants. Toutefois, d'autres hypothèses peuvent probablement expliquer ce taux d'abandon élevé, dont le type et l'étendue des chirurgies, ainsi que la durée moyenne d'hospitalisation, qui est relativement longue chez les participants.

Précisons également que la situation décrite dans la présente étude reflète celle d'un établissement avant la mise en place d'une politique d'évaluation et de gestion de la douleur postopératoire. Comme l'absence de cadre de référence en lien avec l'évaluation de la douleur est fréquente, la situation observée peut néanmoins être représentative de nombreux milieux de soins. L'échantillon de l'étude relativement petit, l'absence de standardisation en lien avec le sexe des participants, le type de chirurgie et les modalités de traitement de la douleur postopératoire peuvent limiter la généralisation des résultats à des contextes cliniques similaires.

Les résultats de la présente étude illustrent bien la problématique exposée dans les publications, selon laquelle l'évaluation et la gestion de la douleur en contexte postopératoire sont sous-optimales $(20,30,44,45)$. Ces résultats sont étonnants puisque l'évaluation clinique du client devrait constituer un élément essentiel de la profession d'infirmière. Cette évaluation devrait, entre autres, inclure le monitorage de la douleur, la surveillance de la sédation après l'administration d'opiacés et d'autres médicaments ayant un effet sur le système nerveux central ainsi que la documentation de l'efficacité des approches thérapeutiques utilisées (46).

Les résultats de la présente étude semblent aussi indiquer que les infirmières utilisent peu les échelles numériques lorsqu'elles évaluent la douleur de leurs patients en contexte postopératoire. Ils indiquent même qu'il n'y a pas de corrélation entre l'intensité de la douleur documentée par l'infirmière et l'intensité de la douleur inscrite par le patient lorsque ceux-ci utilisent des échelles numériques. Pourtant, la Registered Nurse Association of Ontario, l'American Society of PeriAnesthesia Nurses et l'Ordre des infirmières et infirmiers du Québec (OIIQ) recommandaient une évaluation régulière de la douleur à l'aide d'une échelle validée évaluant l'intensité de la douleur, afin de pouvoir assurer une gestion adéquate de la douleur des patients hospitalisés et d'ainsi limiter les effets physiologiques de la douleur $(18,19,46,47)$. Cette sous-utilisation des outils validés pourrait s'expliquer par le fait que, même si les infirmières conviennent de leur valeur, elles restent critiques par rapport à leur objectivité et leur utilité sur le terrain (48). Elles accordent alors davantage d'importance aux paramètres physiologiques et aux comportements non verbaux qu'à l'autoévaluation des patients $(20-22,45,48-51)$.

La méthodologie retenue ne permet pas d'affirmer hors de tout doute que les infirmières évaluent peu et inadéquatement la douleur postopératoire ou même qu'elles sous-estiment la douleur de leur patient. Toutefois, les résultats obtenus indiquent avec certitude une 
documentation insuffisante au dossier du patient. Cette situation peut être problématique, car en l'absence de documentation sur l'évaluation et le traitement de la douleur, il est difficile pour les infirmières et les autres professionnels de la santé d'assurer un suivi adéquat et continu de la douleur (24,25). Il est également difficile de connaître avec exactitude l'efficacité des approches thérapeutiques retenues par l'équipe soignante (25). En l'absence de documentation structurée, claire et concise de la douleur, l'ajustement du plan thérapeutique par le médecin selon l'intensité, la localisation et le type de douleur présente, peut aussi s'avérer ardu (25). Cette situation risque de compromettre l'application des normes recommandées par l'OIIQ en regard de l'évaluation de la douleur et de la surveillance clinique des clients qui reçoivent des médicaments ayant un effet dépresseur sur le système nerveux central (46). Selon ces normes, mises à jour en 2009, la douleur, le degré de sédation et l'état respiratoire doivent être évalués et documentés au moment d'offrir une médication opiacée et lors du pic d'action de cette même médication. Si la médication est prescrite toutes les quatre heures, cela fait minimalement 12 évaluations par jour. Or, dans la présente étude, seulement 2,5\% des patients ont vu leur douleur documentée plus de dix fois lors de la première journée postopératoire. En outre, en raison de l'initiation de la médication opiacée, ces premières doses nécessitent d'autant plus d'évaluations et de surveillance (46).

Les résultats décrits actuellement pourraient aussi s'expliquer par l'importance médicale accordée à l'évaluation de la douleur. Ainsi, si le personnel infirmier a l'impression que la douleur documentée ne sera pas à la base d'une décision thérapeutique, il ne jugera pas nécessaire de la documenter. Comme pour la douleur chronique, il serait important de tenir compte du soulagement de la douleur postopératoire selon une perspective interdisciplinaire, où chaque professionnel peut avoir des conséquences positives et ainsi favoriser le bien-être des patients (52).

Les résultats déclarés dans la présente étude pourraient, entre autres, s'expliquer par un manque de connaissances des infirmières en gestion de la douleur $(20,22,45,53,54)$. En effet, certaines infirmières jugent toujours normal que les patients ressentent de la douleur en postopératoire $(21,27)$ et auraient encore des craintes quant aux risques d'acquérir une dépendance aux opiacés et à leur dangerosité (53-55). Aussi, l'évaluation de la douleur n'est pas encore une des activités prioritaires des infirmières, surtout en présence de contraintes de temps, comme c'est souvent le cas sur une unité de chirurgie $(21,27)$. La majorité des déficits précédents pourraient être grandement attribuables à la crainte d'une dépression respiratoire après l'administration d'opiacés et à une formation théorique inadéquate sur l'évaluation de la douleur, la pharmacologie des analgésiques, les méthodes non pharmacologiques de soulagement de la douleur et les avantages associés au soulagement de la douleur (53-55).

Il est aussi à noter que les croyances des patients auraient également pu influencer les résultats, car, plusieurs patients trouvent normal de ressentir de la douleur après une chirurgie et jugent qu'il n'est pas nécessaire d'en informer le personnel soignant (30). De plus, certains patients ne veulent pas dire qu'ils éprouvent de la douleur ou ont tendance à la sous-estimer lorsque le personnel infirmier l'évalue, car ils croient que le personnel est trop occupé, a des choses plus importantes à faire ou doit s'occuper de patients plus gravement malades $(9,55)$. La crainte et les fausses croyances entourant les opiacés sont aussi à souligner dans l'attitude des patients envers l'expression de leur douleur (29).

\section{CONCLUSION}

À la lumière des résultats de la présente étude et de ce qui a été déclaré dans les publications scientifiques, il est plus que nécessaire de mettre en place des mécanismes de formation du personnel infirmier sur l'évaluation et le soulagement de la douleur postopératoire. Il serait particulièrement important de s'attarder aux fausses croyances des infirmières, de fournir des outils validés et d'en faciliter l'utilisation par les infirmières lors de ces formations. De plus, il faut mettre en place un processus systématique d'évaluation de la douleur et de surveillance de la sédation dans les divers milieux de soins, afin d'amener les infirmières à intégrer l'évaluation et la documentation de la douleur à leur pratique quotidienne et d'en comprendre les bienfaits. Le fait que la douleur ne soit pas une priorité médicale n'encourage pas le personnel infirmier à y accorder de l'importance. Il pourrait aussi être intéressant de sensibiliser les patients à l'importance d'évaluer et de soulager leur douleur en postopératoire lors des rencontres en clinique préopératoire. Le soutien des organisations est aussi primordial pour améliorer la situation. D'ailleurs, de nombreux milieux de soins ont déjà réalisé des initiatives, dont le centre où a eu lieu l'étude, qui a instauré un programme où l'évaluation de la douleur est maintenant considérée comme le cinquième signe vital (51). Des évaluations des répercussions de ces projets sur l'évaluation et le soulagement de la douleur auprès des patients sont en cours. Également, le soulagement de la douleur demeure un travail d'équipe. C'est pourquoi il faudrait préconiser une meilleure collaboration, notamment entre infirmière et médecin, au profit des patients.

REMERCIEMENTS: Nous tenons à remercier les Instituts de recherche en santé du Canada pour le soutien financier, les infirmières du programme de chirurgie et les membres de la direction du centre hospitalier pour leur précieuse collaboration, ainsi que, pour leur aide, madame Nathalie Carrier, statisticienne, monsieur Mathieu Rodrigue, infirmier-clinicien, madame Marie-Hélène Gagné, stagiaire à la collecte de données et madame Émilie Paul-Savoie, assistante professionnelle de recherche. Nous voulons également souligner l'apport de madame Cécile Michaud, professeure agrégée, et de madame Marie-Chantale Loiselle, professeure chargée d'enseignement, pour la révision du manuscrit.

\section{RÉFÉRENCES}

1. Carr DB, Goudas LC. Acute pain. Lancet 1999;353:2051-8.

2. Hallenberg B, Bergbom-Engberg I, Haljamäe H. Patients' experiences of postoperative respirator treatment - influence of anaesthetic and pain treatment regimens. Acta Anaesthesiol Scand 1990;34:557-62.

3. Gélinas C. Le thermomètre d'intensité de douleur : Un nouvel outil pour les patients adultes en soins critiques. Perspective infirmière 2007;4:12.

4. Sjostrom B, Dahlgren LO, Haljamae H. Strategies used in postoperative pain assessment and their clinical accuracy. J Clin Nurs 2000;9:111-8.

5. Nevius KS, D'Arcy Y. Decrease recovery time with proper pain management. OR Nurse 2008;2:34-40.

6. Puntillo KA, Weiss SJ. Pain: its mediators and associated morbidity in critically ill cardiovascular surgical patients. Nurse Res 1994:43:31-6.

7. Carr EC, Thomas N, Wilson-Barnett J. Patient experiences of anxiety, depression and acute pain after surgery: a longitudinal perspective. Int J Nurs Stud 2004;42:521-30.

8. Dunwoody CJ, Krenzischek DA, Pasero C, Rathmell JP, Polomano RC. Assessment, physiological monitoring, and consequences of inadequately treated acute pain. Pain Manage Nurs 2008;9:S11-21.

9. Mackintoch C. Assessment and management of patients with post-operative pain. Nursing standard 2007;22:49-55.

10. Morrison RS, Magaziner J, McLaughlin MA et coll. The impact of post-operative pain on outcomes following hip fracture. Pain 2003;103:303-11.

11. Lynch EP, Lazor MA, Gellis JE, Orav J, Goldman L, Marcantonio ER. The impact of postoperative pain on the development of postoperative delirium. Anesth Analg 1998;86:781-5.

12. Carr E, Thomas VJ. Anticipating and experiencing post-operative pain: the patients' perspective. J Clin Nurs 1997;6:191-201.

13. Callesen T, Bech K, Kehlet H. Prospective study of chronic pain after groin hernia repair. Br J Surg 1999;86:1528-31.

14. Kalso E, Perttunen K, Kaasinen S. Pain after thoracic surgery. Acta Anaesthesiol Scand 1992;36:96-100. 
15. Katz J, Jackson M, Kavanagh BP, Sandler AN. Acute pain after thoracic surgery predicts long-term post-thoracotomy pain. Clin J Pain 1996;12:50-5.

16. Perkins FM, Kehlet H. Chronic pain as an outcome of surgery. A review of predictive factors. Anesthesiology 2000;93:1123-33.

17. Tasmuth T, Kataja M, Blomqvist C, von Smitten K, Kalso E. Treatment-related factors predisposing to chronic pain in patients with breast cancer - a multivariate approach. Acta Oncol 1997;36:625-30.

18. Registered Nurses Association of Ontario (RNAO). Nursing Best Practice Guideline - Assessment and Management of Pain. Toronto: RNAO, 2002.

19. American Society of PeriAnesthesia Nurses (ASPAN), ed. ASPAN pain and comfort guidelines, 2003.

20. McCaffery M, Pasero C, Ferrell BR. Nurses' decisions about opioid dose. Am J Nurs 2007;107:35-9.

21. Manias E, Bucknall T, Botti M. Assessment of patient pain in the postoperative context. West J Nurs Res 2004;26:751-69.

22. Comley AL, Banks JC. Pain management: clinician survey and institutional needs assessment. Proc (Bayl Univ Med Cent) 2000;13:230-5.

23. Gunningberg L, Idvall E. The quality of postoperative pain management from the perspectives of patients, nurses and patient records. Journal of nursing management 2007;15:756.

24. Dalton JA, Carlson J, Blau W, Lindley C, Greer SM, Youngblood R. Documentation of pain assessment and treatment: how are we doing? Pain Manage Nurs 2001;2:54-64.

25. Malek CJ, Olivieri RJ. Pain management: documenting the decision making process. Nurs Case Manage 1996;1:64-74.

26. Manias E. Medication trends and documentation of pain management following surgery. Nurs Health Sci 2003;5:85-94

27. Manias E, Botti M, Bucknall T. Observation of pain assessment and management - the complexities of clinical practice. J Clin Nurs 2002;11:724-33.

28. Idvall E, Hamrin E, Sjöström B, Unosson M. Patient and nurse assessment of quality of care in postoperative pain management. Qual Saf Health Care 2002;11:327-34.

29. Botti M, Bucknall T, Manias E. The problem of postoperative pain: issues for future research. Int J Nurs Pract 2004;10:257-63.

30. Hutchison RW. Challenges in acute post-operative pain management. Am J Health Syst Pharm 2007;64:S2-5.

31. Choinière M, Melzack R, Girard N, Rondeau J, Paquin M. Comparisons between patients' and nurses' assessments of pain and medication efficacy in severe burn injuries. Pain 1990;40:143-52.

32. Lamb J, Ross S. Pain management: a patient's perspective. Can Nurse 1999;95:30-3.

33. Watt-Watson J, Stevens B. Managing pain after coronary artery bypass surgery. J Cardiovasc Nurs 1998;12:39-51.

34. Ordre des infirmières et infirmiers du Québec (OIIQ). Avis sur la surveillance clinique des clients qui reçoivent des médicaments ayant un effet dépressif sur le système nerveux central (SNC). Montréal, 2004.

35. Gerbershagen HJ, Ozgür E, Straub K, et al. Prevalence, severity, and chronicity of pain and general health-related quality of life in patients with localized prostate cancer. Eur J Pain 2008;12:339-50.
36. Yermal SJ. Perioperative pain, psychological distress and immune function in men throughout the radical retropubic prostatectomy experience 2005:205.

37. Marchand S, Charest J, Li J, Chenard JR, Lavignolle B, Laurencelle L. Is TENS purely a placebo effect? A controlled study on chronic low back pain. Pain 1993;54:99-106.

38. Bourgault P, Michaud C, Bolduc N, Lapré J. La gestion de la douleur comme pratique exemplaire en soins infirmiers, 2009.

39. Bourgault P, Devroede G, St-Cyr-Tribble D, Marchand SJ. Help-seeking process in women with irritable bowel syndrome. Part 1: study results. Gastrointest Nurs 2008;6:24-31.

40. Tousignant-Laflamme Y, Marchand S. Sex differences in cardiac and autonomic response to clinical and experimental pain in LBP patients. European Journal of Pain 2006;10:603-14.

41. Leduc G. Rôle des odeurs sur la perception de la douleur, 2007.

42. Grondin F, Bourgault P, Bolduc N. L'impact d'un modèle de soin centré sur la famille dans la gestion de la douleur chez une clientèle âgée ayant subi une chirurgie de la hanche, 2009.

43. Price DD, McGrath PA, Rafii A, Buckingham B. The validation of visual analogue scales as ratio scale measures for chronic and experimental pain. Pain 1983;17:45-56.

44. Apfelbaum JL, Chen C, Mehta SS, Gan TJ. Postoperative pain experience: results from a national survey suggest postoperative pain continues to be undermanaged. Anesth Analg 2003;97:534.

45. McCaffery M, Ferrell BR, Pasero C. Nurses' personal opinions about patients' pain and their effect on recorded assessments and titration of opioid doses. Pain Manage Nurs 2000;1:79-87.

46. Ordre des infirmières et infirmiers du Québec (OIIQ). Surveillance clinique des clients qui reçoivent des médicaments ayant un effet dépressif sur le système nerveux central. Montréal: OIIQ, 2009:24.

47. Krenzischek DA, Wilson L. An introduction to the ASPAN pain and comfort clinical guideline. Journal of PeriAnesthesia Nursing 2003;18:228-36.

48. Young JL, Horton FM, Davidhizar R. Nursing attitudes and beliefs in pain assessment and management. J Adv Nurs 2006;53:412-21.

49. Clarke EB, French B, Bilodeau ML, Capasso VC, Edwards A, Empoliti J. Pain management knowledge, attitudes and clinical practice: the impact of nurses' characteristics and education. J Pain Symptom Manage 1996;11:18-31.

50. Manias E, Bucknall T, Botti M. Nurses' strategies for managing pain in the postoperative setting. Pain Manage Nurs 2005;6:18-29.

51. Merboth MK, Barnason S. Managing pain: the fifth vital sign. Nurs Clin North Am 2000;35:375-83.

52. Stanos S, Houle TT. Multidisciplinary and interdisciplinary management of chronic pain. Phys Med Rehabil Clin North Am 2006;17:435-50.

53. Coulling S. Nurses' and doctors' knowledge of pain after surgery. Nurs Stand 2005;19:41-9.

54. Matthews E, Malcolm C. Pain management. Nurses' knowledge and attitudes in pain management practice. $\mathrm{Br} \mathrm{J}$ Nurs 2007;16:174-9.

55. Gray A. Barriers to effective pain management. London: Whurr Publishers, 2005:113-28. 


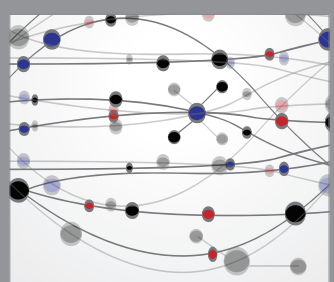

The Scientific World Journal
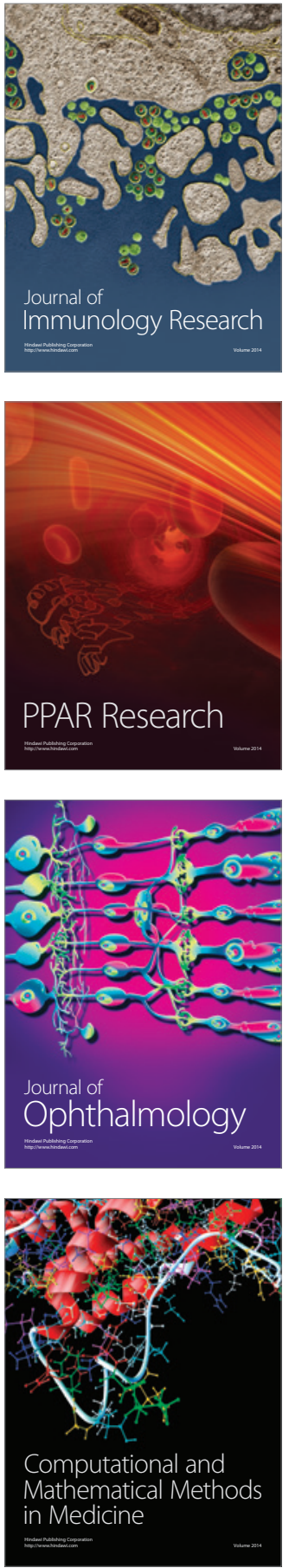

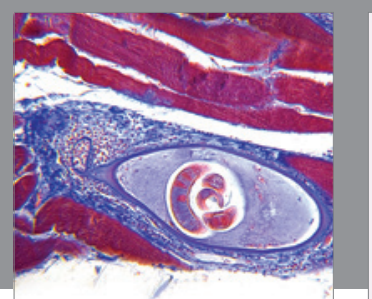

Gastroenterology Research and Practice

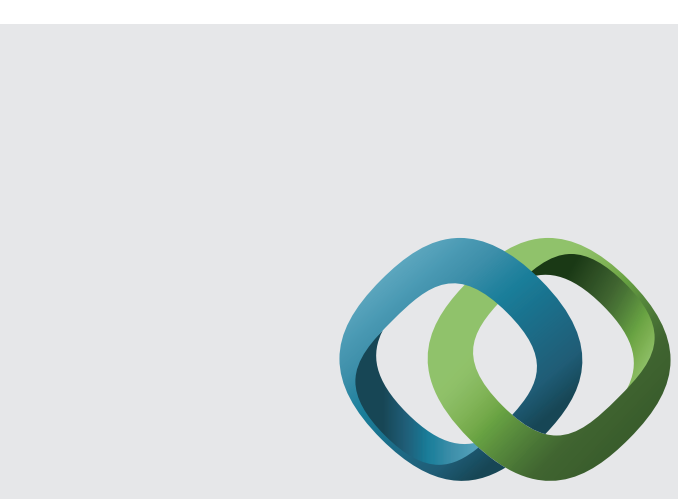

\section{Hindawi}

Submit your manuscripts at

http://www.hindawi.com
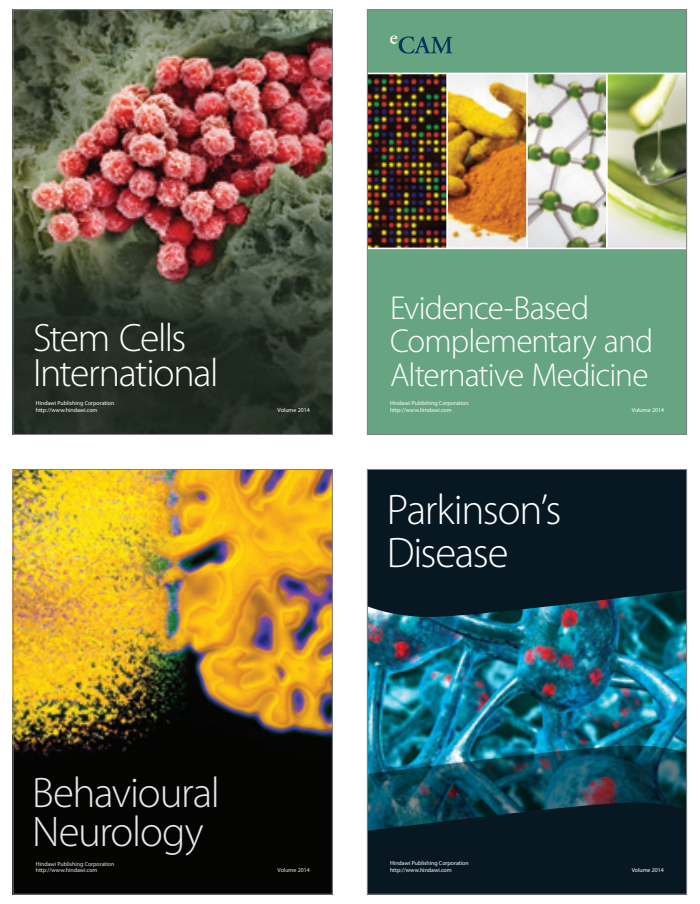
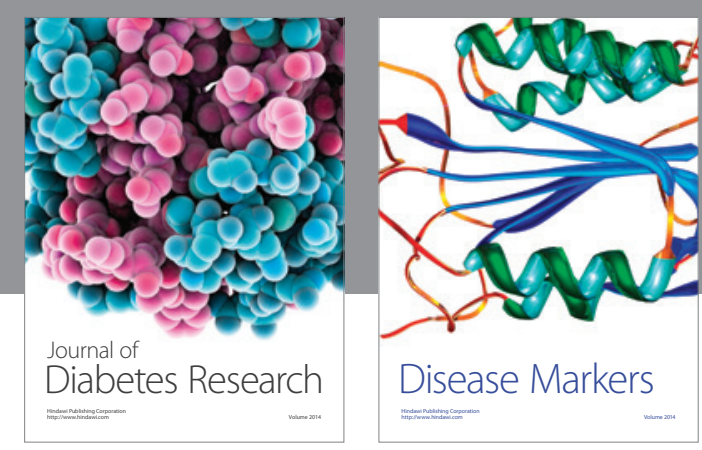

Disease Markers
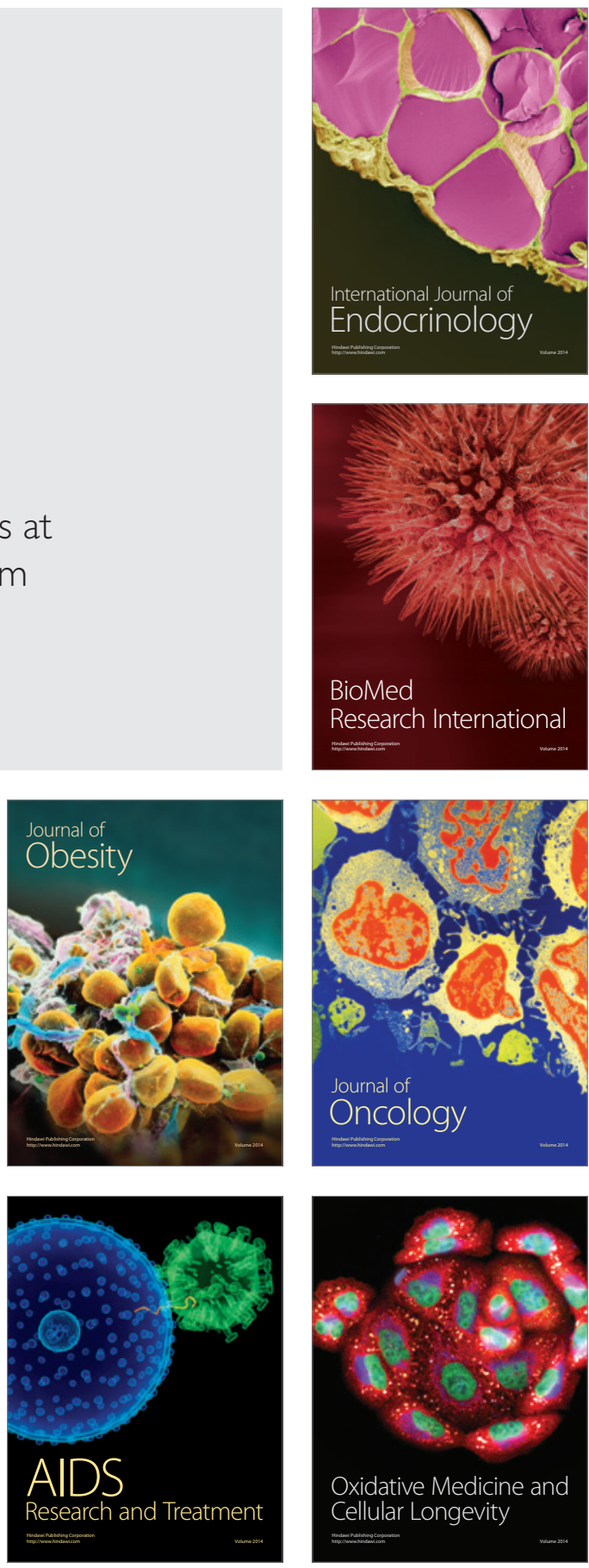